The NCCN Oncology Research Program (ORP) strives to improve the quality of life for patients and reduce cancer-related deaths by advancing cancer therapies through research. Since the program's establishment in 1999, the NCCN ORP has brought millions of dollars in research grants to investigators at NCCN Member Institutions. Research grants are provided to NCCN through collaborations with pharmaceutical and biotechnology companies; these grants are in turn used to support scientifically meritorious cancer research efforts.

NCCN ORP studies typically explore new avenues of clinical investigation and seek answers to important cancer-related questions. All studies are approved and funded through a scientific peer-review process and are overseen by the ORP.

This feature highlights an NCCN study funded through the grant mechanism.

doi: 10.6004/jnccn.2020.0004

For more information on specific trials, including patient selection criteria, use the contact information listed with each study.

For more information on the NCCN ORP, including a complete detailing of the clinical studies currently underway at NCCN Member Institutions, go to www. NCCN.org/clinical_trials/clinicians.asp.

\section{Phase Ib Dose Escalation Trial of Carfilzomib in Combination With Bendamustine and Rituximab in Patients With Relapsed or Refractory Non-Hodgkin Lymphoma}

Principal Investigator: Charalambos Andreadis, MD, MSCE Condition: Relapsed or refractory non-Hodgkin lymphoma Institution: UCSF Helen Diller Family Comprehensive Cancer Center

This study is being conducted as a phase $\mathrm{lb}$, open-label, nonrandomized, single-institution study to evaluate the safety and tolerability of carfilzomib in combination with bendamustine and rituximab in patients with relapsed or refractory non-Hodgkin lymphoma ( $\mathrm{NHL}_{\text {; }}$ lymphoplasmacytic, marginal zone, follicular, mantle cell, diffuse large $\mathrm{B}$ cell) and to determine the recommended phase II dose and preliminary efficacy of this combination. The study will have 2 phases: a dose-escalation phase to determine the maximal tolerated dose (MTD) of carfilzomib in this combination in which participants will be monitored for toxicity, tolerability, and response, and a dose-expansion phase that will determine the preliminary efficacy in patients with mantle cell lymphoma or any other disease subtype in which there is a preliminary efficacy signal observed.

Determination of the MTD will follow standard $3+3$ design with escalation of the carfilzomib dose only. Dose levels of carfilzomib will be $15 \mathrm{mg} / \mathrm{m}^{2}, 36 \mathrm{mg} / \mathrm{m}^{2}, 56 \mathrm{mg} / \mathrm{m}^{2}$, and $70 \mathrm{mg} / \mathrm{m}^{2}$ intravenously administered on days 2,9 , and 16 . The last 3 cohorts will have a starting carfilzomib dose of $20 \mathrm{mg} / \mathrm{m}^{2}$ on days 1 and 2 . Bendamustine will be administered at the well-tolerated dose of $90 \mathrm{mg} / \mathrm{m}^{2}$ intravenously on days 1 and 2. A dose de-escalation of bendamustine will occur if the starting dose proves intolerable in this combination. Rituximab will be given at a dose of $375 \mathrm{mg} / \mathrm{m}^{2}$ on day 9 of cycle 1 and day 1 of subsequent cycles.

\section{Primary Objective:}

- Assess the safety and tolerability of carfilzomib when combined with bendamustine and rituximab in patients with relapsed or refractory $\mathrm{NHL}$

\section{Secondary Objective:}

- Evaluate the preliminary antitumor activity of carfilzomib with bendamustine and rituximab in patients with $\mathrm{NHL}$ (dose escalation) and with specific NHL subtypes (dose expansion)

\section{Exploratory Objectives:}

- Describe overall survival in patients treated with these agents

- Evaluate the relationship between antitumor activity or toxicity of carfilzomib and bendamustine with markers of activation of the terminal unfolded protein response and/ or modulation of the apoptotic pathway in primary tumor tissue 
Contacts: Charalambos Andreadis, MD • 415-353-8363 •

candreadis@medicine.ucsf.edu

Erika Cavallone • 415-476-4765 •

Erika.Cavallone@ucsf.edu

Charlie Aoun • 415-502-1564 •

Charlie.Aoun@ucsf.edu

ClinicalTrials.gov Identifier: NCT02187133

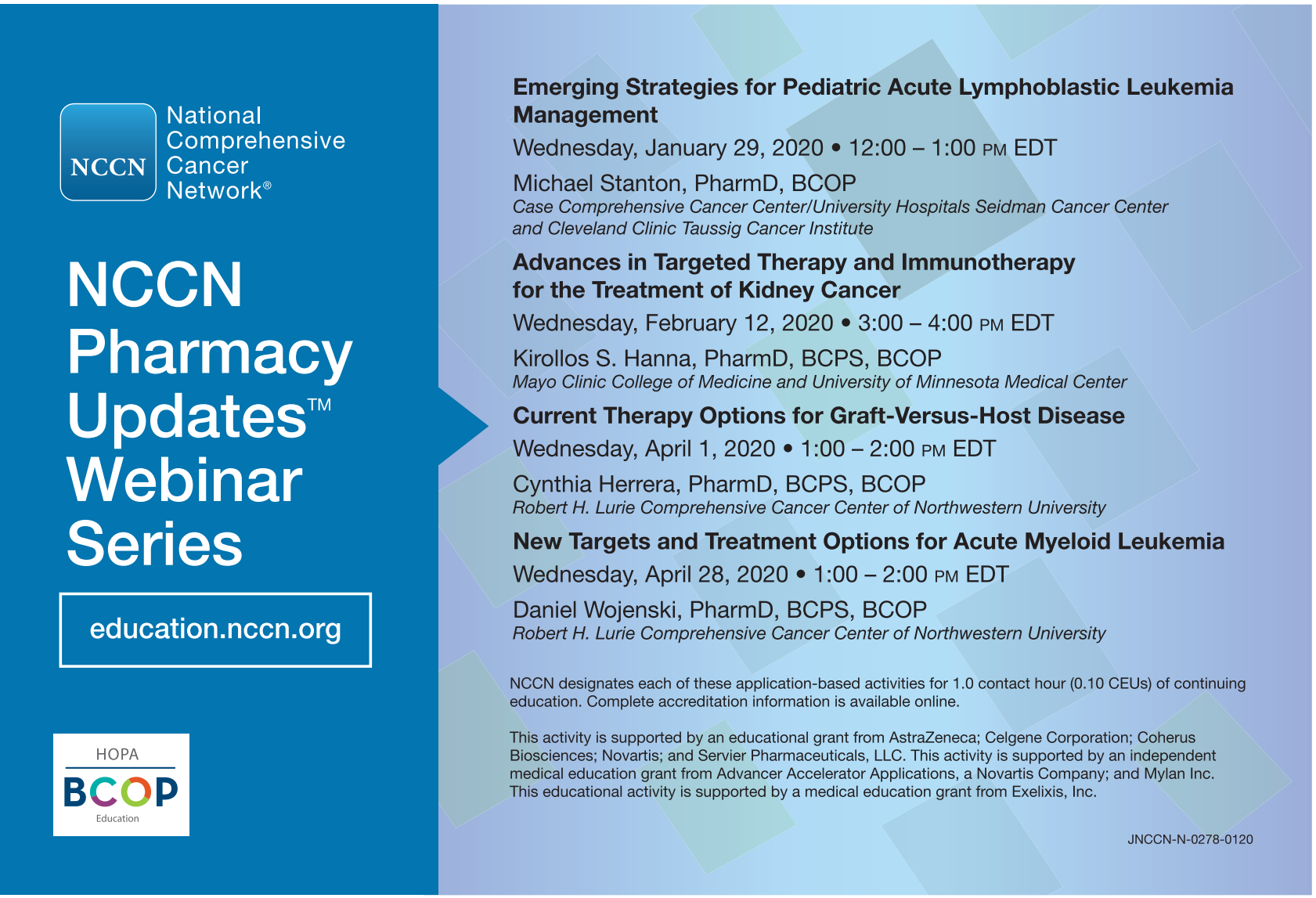

